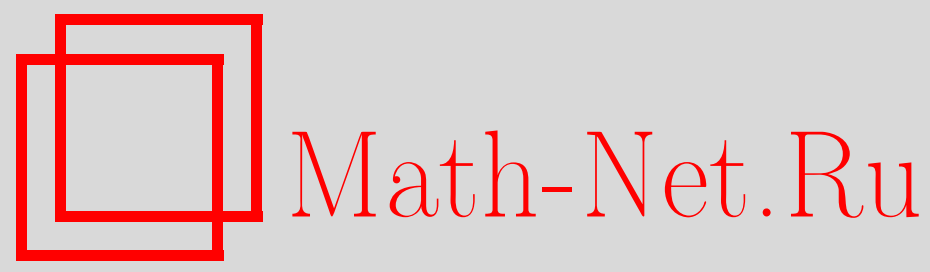

А. Д. Мышкис, О полулинейных обобщенно канонических системах уравнений с частными производными первого порядка гиперболического типа, Матем. заметки, 2002, том 72, выпуск 5, 729-738

DOI: https://doi.org/10.4213/mzm462

Использование Общероссийского математического портала Math-Net.Ru подразумевает, что вы прочитали и согласны с пользовательским соглашением http://www . mathnet.ru/rus/agreement

Параметры загрузки:

IP : 3.80 .181 .102

26 апреля 2023 г., 15:25:43

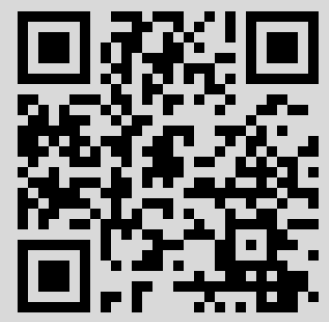




\title{
О ПОЛУЛИНЕЙНЫХ ОБОБЩЕННО КАНОНИЧЕСКИХ СИСТЕМАХ УРАВНЕНИЙ С ЧАСТНЫМИ ПРОИЗВОДНЫМИ ПЕРВОГО ПОРЯДКА ГИПЕРБОЛИЧЕСКОГО ТИПА
}

\section{А. Д. Мышкис}

\begin{abstract}
С помощью метода характеристик доказаны теоремы о непрерьвной разрешимости и о свойствах решений начально-граничной задачи для обобщенно канонической системы полулинейных уравнений с частными производными первого порядка гиперболического типа в области общего вида $(m+1)$-мерного пространства.

Библиограффия: 5 названий.
\end{abstract}

1. Введение. Полулинейная каноническая система уравнений с частными производными гиперболического типа и единственной пространственной переменной имеет обший вид

$$
\frac{\partial u_{i}(x, t)}{\partial t}+\lambda_{i}(x, t) \frac{\partial u_{i}(x, t)}{\partial x}=f_{i}(x, t, u(x, t)), \quad i=1, \ldots, n,
$$

где $u=\left(u_{1}, \ldots, u_{n}\right)$. Как известно, при постановке начально-граничных задач для этих систем и при исследовании их решений фундаментальную роль играет метод характеристик (см., например, [1], [2], где имеются дальнейшие ссылки). Напомним, что характеристиками $i$-го семейства $(i=1, \ldots, n)$ системы уравнений (1) называются интегральные линии обыкновенного дифференциального уравнения (ОДУ) $d x(t) / d t=$ $\lambda_{i}(x(t), t)$ в плоскости $x, t$. После интегрирования $i$-го уравнения (1) вдоль характеристик $i$-го семейства для $i=1, \ldots, n$ получается система интегро-функциональных уравнений типа Вольтерра. На этом переходе и основан метод характеристик.

Аналогичный метод для систем уравнений в $\mathbb{R}^{m+1}(m \geqslant 1)$, записанных в форме, которую мы назвали обобщенной канонической:

$$
\frac{\partial u_{i}(x, t)}{\partial t}+\sum_{j=1}^{m} \lambda_{i j}(x, t) \frac{\partial u_{i}(x, t)}{\partial x_{j}}=f_{i}(x, t, u(x, t)), \quad i=1, \ldots, n,
$$

где $n \geqslant 1, x:=\left(x_{1}, \ldots, x_{m}\right), u:=\left(u_{1}, \ldots, u_{n}\right)$, изучен гораздо меньше. В работах М. Чинквини-Чибрарио и Л. Чезари (см. [3], [4], где имеются дальнейшие ссылки) этот метод применялся к доказательству разрешимости задачи Коши и сходных задач для системы уравнений (2) и некоторых более общих (в том числе квазилинейных) систем

Работа выполнена при поддержке Российского фонда фундаментальных исследований, грант № 00-01-00683a, и Фонда фундаментальных исследований Министерства путей сообщения РФ. 
уравнений. При этом характеристики $i$-го семейства $(i=1, \ldots, n)$ - это интегральные линии системы ОДУ $d x_{j}(t) / d t=\lambda_{i j}(x(t), t), j=1, \ldots, m$, или, короче,

$$
\frac{d x(t)}{d t}=\lambda_{i}(x, t),
$$

где обозначено $\lambda_{i}(x, t):=\left(\lambda_{i 1}(x, t), \ldots, \lambda_{i m}(x, t)\right)$. Вдоль такой характеристики левую часть системы (2) можно записать в виде $d u_{i}(t) / d t$, что дает возможность перейти $\mathrm{k}$ равносильной системе интегральных уравнений типа Вольтерра.

Задача с начальньми и граничными условиями для общей системы уравнений (2), по-видимому, не только не изучалась, но даже не ставилась. Целью настоящей статьи является постановка этой задачи и доказательство основных теорем о ее разрешимости. Мы ограничимся непрерьвньми обобщенными решениями (определение см. в п. 3), оставив рассмотрение гладких решений и разрывных решений для дальнейших исследований. Отметим, что задача Коши для системы уравнений (2) является частньп случаем рассматриваемой здесь общей задачи.

2. Общая постановка задачи. В основу мы положим некоторое непустое замкнутое множество $\Pi \subset \mathbb{R}^{m+1}$, в котором будут происходить все события. Функции $\lambda_{i j}$ впредь предполагаются непрерывными в некоторой окрестности множества П и такими, что в этой окрестности имеет место единственность продолжения решений каждой из $n$ систем уравнений (3) в направлении убьвания $t$, причем при этом продолжении соответствующая интегральная линия не может все время принадлежать П. Обозначим такое решение для $i$-й системы $(3)$ с конечной точкой $\left(x^{0}, t^{0}\right) \in \Pi$ (т.е. $t^{0}=\max t$ для этого решения) через $x=\varphi_{i}\left(t ; x^{0}, t^{0}\right)\left(t \leqslant t^{0}\right)$ и положим

$$
\begin{gathered}
\chi_{i}\left(x^{0}, t^{0}\right):=\sup \left\{t<t^{0}: \varphi_{i}\left(t ; x^{0}, t^{0}\right) \notin \Pi\right\}, \\
\Phi_{i}\left(x^{0}, t^{0}\right):=\left(\varphi_{i}\left(\chi_{i}\left(x^{0}, t^{0}\right) ; x^{0}, t^{0}\right), \chi_{t}\left(x^{0}, t^{0}\right)\right) .
\end{gathered}
$$

Ясно, что $\Phi_{i}\left(x^{0}, t^{0}\right) \in \partial$. График этого решения при $\chi_{i}\left(x^{0}, t^{0}\right) \leqslant t<t^{0}$ назовем характеристикой ( $i$-го семейства).

Естественные добавочные условия к системе уравнений (2) вьглядят следующим образом:

$$
u_{i}(x, t)=g_{i}(x, t, u(x, t)), \quad i \in I(x, t), \quad(x, t) \in \partial \Pi,
$$

где $I(x, t)$ есть множество индексов $i \in I:=\{1, \ldots, n\}$, для которых $\chi_{i}(x, t)=t$. Каждая функция $g_{i}$ должна быть задана на множестве $S_{i} \times \mathbb{R}^{n}$, где $S_{i}=\{(x, t) \in \partial \Pi$ : $\left.\chi_{i}(x, t)=t\right\}$, зависеть только от аргументов $x, t$ и $u_{j}(x, t): j \in I^{\prime}(x, t):=I \backslash I(x, t)$. Таким образом, на каждом непустом множестве $S_{i_{1}} \cap \cdots \cap S_{i_{k}}$ каждая из функций $g_{i_{j}}$ не должна зависеть от аргументов $u_{i_{1}}, \ldots, u_{i_{k}}$. В частности, в точках $(x, t) \in \partial \Pi$, для которых $I(x, t)=\varnothing$, т.е. $(x, t) \in \partial \Pi \backslash \cup_{i} S_{i}$, условие (4) не ставится. Если $I(x, t)=I$, т.е. $(x, t) \in S:=\cap_{i} S_{i}$, то условия (4) приобретают вид $u_{i}(x, t)=g_{i}(x, t), i \in I,-$ это начальные условия. Если же $\varnothing \subset I(x, t) \subset I$ ( $\subset$ понимается как строгое включение), то условия (4) естественно трактовать как краевые (граничные). Таким образом, система уравнений (2) вместе с добавочньми условиями (4) представляет собой начально-граничную (смешанную) задачу, причем задача Коши рассматривается здесь как частный случай (она получается, если $\forall(x, t) \in \partial \Pi \Rightarrow I(x, t) \in\{\varnothing, I\})$.

Если решение $u$ задачи (2), (4) непрерьвно дифференцируемо в окрестности П, то интегрируя $i$-е уравнение $(2)$ при $(x, t) \in$ П вдоль любой характеристики $i$-го семейства, 
а затем, применяя условие (4), приходим к системе интегро-функциональных уравнений типа Вольтерра:

$$
\begin{gathered}
u_{i}(x, t)=g_{i}\left(\Phi_{i}(x, t), u\left(\Phi_{i}(x, t)\right)\right)+\int_{\chi_{i}(x, t)}^{t} f_{i}\left(\varphi_{i}(\tau ; x, t), \tau, u\left(\varphi_{i}(\tau ; x, t), \tau\right)\right) d \tau, \\
i \in I, \quad(x, t) \in \Pi .
\end{gathered}
$$

Обратно, если функция $u$, непрерьвно дифференцируемая в окрестности П, удовлетворяет системе уравнений (5), то она, очевидно, является решением задачи (2), (4). Это дает основание для того, чтобы функцию $u: \Pi \rightarrow \mathbb{R}^{n}$, удовлетворяющую системе уравнений (5), назьвать обобщенным решением задачи (2), (4), даже если она не является непрерывно дифференцируемой, лишь бы интеграл в правой части уравнения (5) имел смысл.

Обобщенное решение $u$ задачи (2), (4) может быть определено не на всем множестве $\Pi$, а только на каком-либо множестве $K \subseteq \Pi$. От $K$ надо требовать, чтобы для любых $\left(x^{0}, t^{0}\right) \in K, i \in I$, вся характеристика $i$-го семейства с конечной точкой $\left(x^{0}, t^{0}\right)$ принадлежала $K$, причем определение обобщенного решения $u: K \rightarrow \mathbb{R}^{n}$ задачи (2), (4) как решения системы уравнений (5) не меняется. Непустое множество $K$, удовлетворяющее указанным требованиям, будем называть допустимым. Ясно, что объединение или непустое пересечение любой совокупности допустимых множеств также является допустимым множеством; если множество $K$ допустимое, то и любое непустое множество вида $\{(x, t) \in K: t \leqslant$ const $\}$ допустимое.

3. Непрерывное обобщенное решение. Непрерывным обобщенным решением задачи $(2),(4)$ будем называть непрерывную функцию $u$, определенную на допустимом множестве $K=K_{u}$ и удовлетворяющую в каждой точке $K$ системе уравнений (5). В дальнейшем для краткости слово "обобщенное" будем опускать.

При рассмотрении непрерьвных решений естественно наложить следующее условие на расположение семейства характеристик относительно множества П.

У. Все функиии $\chi_{i}: \Pi \rightarrow \mathbb{R}(i \in I ;$ см. n. 2) непрерывные.

Из этого условия сразу следует, что все множества $S_{i}$ (см. п. 2) замкнутые. Кроме того, условие У ограничивает класс рассматриваемых множеств П. Например, множество П при условии У не может иметь ограниченных изолированных "пустот"; более точно, справедлива следующая теорема.

Теорема 1. Если выполнено условие У и множество П связное, то множество $\mathbb{R}^{m+1} \backslash \Pi$ не может иметь ограниченных компонент связности, удаленных на полохительное расстояние от своего дополнения в $\mathbb{R}^{m+1} \backslash \Pi$.

ДокАЗАТЕЛЬСтво. Пусть $D$ - такая компонента и $(\underline{x}, \underline{t})($ соответственно $(\bar{x}, \bar{t}))-$ какая-либо из точек $\partial D$ с наименьшим (наибольшим) возможным значением $t$. Ясно, что $\Phi_{1}(\underline{x}, \underline{t}) \notin \partial D$. Из связности множеств $D$ и П следует, что и множество $\partial D$ связное, а потому и множество $\Phi_{1}(\partial D)$ как непрерывный образ связного множества также связно. Так как оно содержится в $\partial \Pi$, то $\Phi_{1}(\partial D) \cap \partial D=\varnothing ;$ значит, и $\Phi_{1}(\bar{x}, \bar{t}) \notin \partial D$.

Выберем $\varepsilon>0$ таким, чтобы интегральная воронка $W$ системы (3) для $i=1 \mathrm{c}$ вершиной в точке $(\bar{x}, \bar{t})$ целиком при $\bar{t}<t \leqslant \bar{t}+\varepsilon$ принадлежала $\Pi \backslash \partial \Pi$. Тогда, если точка $(\tilde{x}, \tilde{t}) \in D$ находится достаточно близко от $(\bar{x}, \bar{t})$, то интегральная воронка $\widetilde{W}$ той 
же системы с вершиной в точке $(\tilde{x}, \tilde{t})$ при $\tilde{t} \leqslant t \leqslant \bar{t}+\varepsilon$ целиком расположена как угодно близко от $W$ и потому принадлежит $D \cup$ П. Возьмем какую-либо характеристику системы (3) с $i=1$ и концевой точкой $\left(x^{0}, \bar{t}+\varepsilon\right) \in \widetilde{W}$. Тогда $\Phi_{1}\left(x^{0}, \bar{t}+\varepsilon\right) \in \widetilde{W}$ и потому $\Phi_{1}\left(x^{0}, \bar{t}+\varepsilon\right) \in \partial D$. Переход к пределу при $\varepsilon \rightarrow 0$ приводит к противоречию $\mathrm{c}$ доказанным вьше.

Теорема 1 доказана.

ЗАмЕчАНИЕ. Несколько усложняя доказательство, можно проверить, что требование связности множества П в формулировке теоремы 1 является излишним.

Отметим для дальнейшего, что если условие У выполнено в П, то оно вьполнено и в каждом допустимом множестве $K \subset \Pi$, а также в каждом множестве вида $\{(x, t) \in \Pi$ : $t \geqslant$ const $\}$. Сужение непрерывного решения $u$ задачи $(2),(4)$ в каком-либо допустимом множестве $K$ на непустое множество $K:=\left\{(x, t) \in K: t \geqslant t_{0}\right\}$ является непрерьвньп решением этой же задачи в $K$, если недостающие начальные данные при $t=t_{0}$ задаются самим решением $u$.

4. Теорема о локальной непрерывной разрешимости задачи (2), (4). Назовем иепочкой любую последовательность попарно различных точек $\left(x^{j}, t^{j}\right) \in \Pi$, $j=1, \ldots, k(\geqslant 1)$, для которой $\left(x^{j}, t^{j}\right) \in\left\{\Phi_{1}\left(x^{j-1}, t^{j-1}\right), \ldots, \Phi_{n}\left(x^{j-1}, t^{j-1}\right)\right\}, j=$ $2, \ldots, k$; назовем $k$ длиной такой цепочки, а $\left(x^{1}, t^{1}\right)$ - ее концом. Через $U(A, \varepsilon)$ будем обозначать $\varepsilon$-окрестность множества $A$.

Tеорема 2. Пусть все функиии $\left[(x, t, u) \mapsto f_{i}(x, t, u)\right]: \Pi \times \mathbb{R}^{n} \rightarrow \mathbb{R}$ непрерывньи $u$, как и функции $\left[(x, t, u) \mapsto g_{i}(x, t, u)\right]: S_{i} \times \mathbb{R}^{n} \rightarrow \mathbb{R}$, локально ограниченьи $u$ локально удовлетворяют по и условию Липшица. Пусть, далее, выполнено условие У и длины всех иепочек локально равномерно ограничены. Наконеи, пусть для некоторой точки $\left(x^{0}, t^{0}\right) \in S$ выполнены условия:

$\mathrm{Y}_{1}$. для любого $\varepsilon>0$ существуют такие $\delta>0$ и открытое в П допустимое множсество $K$, что

$$
U\left(\left(x^{0}, t^{0}\right), \delta\right) \cap \Pi \subseteq K \subseteq U\left(\left(x^{0}, t^{0}\right), \varepsilon\right)
$$

$\mathrm{y}_{2}$. для любих допустимого множества $K$ и функиий $u_{i} \in C\left(S_{i} \cap K, \mathbb{R}^{n}\right), i \in I$, удовлетворяющих в рывны в $S_{i} \cap K, i \in I$;

$\mathrm{y}_{3 .}$ в $C\left(\partial П, \mathbb{R}^{n}\right)$ существует функиия, удовлетворяющая условиям (4).

Тогда существуют $\delta>0$, открытое в П допустимое мнохсество $K \supseteq U\left(\left(x^{0}, t^{0}\right)\right.$, $\delta) \cap \Pi$ и непрерывное решение $u: K \rightarrow \mathbb{R}^{n}$ задачи (2), (4). Это решение единственно на всяком допустимом множестве $\widetilde{K}$, для которого $\left(x^{0}, t^{0}\right) \in \widetilde{K} \subseteq K$.

ДокАЗАТЕЛЬСтво. Зададим произвольно $\varepsilon>0$ и возьмем в качестве $K=K_{\varepsilon}$ какое-либо допустимое множество, о котором говорится в условии У $\mathrm{y}_{1}$. Определим на множестве функций из $C\left(K, \mathbb{R}^{n}\right)$, удовлетворяюших в $\partial \Pi \cap K$ условиям (4), отображение $P=P_{\varepsilon}$, задаваемое правьги частями системы уравнений (5):

$$
\begin{gathered}
(P u)_{i}(x, t):=g_{i}\left(\Phi_{i}(x, t), u\left(\Phi_{i}(x, t)\right)\right)+\int_{\chi_{i}(x, t)}^{t} f_{i}\left(\varphi_{i}(\tau ; x, t), \tau, u\left(\varphi_{i}(\tau ; x, t), \tau\right)\right) d \tau \\
i \in I, \quad(x, t) \in K .
\end{gathered}
$$


Из непрерывной зависимости решений системы (3) от их конечной точки и из условий У и У 2 следует, что $P$ отображает это множество в себя. Кроме того, очевидно, что $\left[(x, t) \in S \cap K, u \in C\left(K, \mathbb{R}^{n}\right)\right] \Rightarrow(P u)(x, t)=g(x, t)$.

Проверим, далее, что для любого достаточно большого числа $M$ справедливо неравенство $\left\|P^{r-1} u\right\| \leqslant M$, если $u \in C\left(K, \mathbb{R}^{n}\right),\|u\| \leqslant M, \varepsilon$ достаточно мало́, а $r-$ максимальная длина цепочек в $K$. (Под $\|\cdot\|$ будем понимать для простоты норму в пространстве $C\left(K, \mathbb{R}^{n}\right)$, основанную на норме $|x|:=\max _{i}\left|x_{i}\right|$ векторов. Кроме того, впредь будем считать, что $r \geqslant 2$, так как при $r=1$ утверждение теоремы 2 сразу вытекает из ее предположений.) Для этого обозначим через $K_{j}(j=1, \ldots, r)$ множество точек $(x, t) \in K$, для которых длины цепочек с конщом в $(x, t)$ не превосходят $j$. Ясно, что $S \cap K=K_{1} \subseteq K_{2} \subseteq \cdots \subseteq K_{r}=K$.

Если $(x, t) \in K_{1}$, то $|(P u)(x, t)| \leqslant p_{1}$, где $p_{1}$ - верхняя грань функции $|g|$ на $S \cap K$. Если же $(x, t) \in K_{2}$, то в силу формулы (6) и локальной ограниченности функций $f_{i}$ получаем, что $|(P u)(x, t)| \leqslant p_{1}+O(\varepsilon)$, причем оценка $O(\varepsilon)$ здесь и далее равномерна по всем точкам $(x, t)$ и всем функциям $u \in C(K, \mathbb{R})$, ограниченных по норме какой-либо единой константой.

Пусть теперь $(x, t) \in K_{3}$. Тогда из формулы (6) получаем

$$
\left|\left(P^{2} u\right)(x, t)\right|=\max _{j}\left|g_{j}\left(\Phi_{j}(x, t),(P u)\left(\Phi_{j}(x, t)\right)\right)\right|+O(\varepsilon),
$$

где все $\Phi_{j}(x, t) \in K_{2}$. Представив $(P u)\left(\Phi_{j}(x, t)\right)$ с помощью формулы $(6)$ и воспользовавшись условием Липшица для $g_{j}$, получаем

$$
\begin{aligned}
& \left|g_{j}\left(\Phi_{j}(x, t),(P u)\left(\Phi_{j}(x, t)\right)\right)\right| \\
& \quad=\left|g_{j}\left(\Phi_{j}(x, t), \ldots, g_{i}\left(\Phi_{i}\left(\Phi_{j}(x, t), u\left(\Phi_{1}\left(\Phi_{j}(x, t)\right)\right)\right)\right), \ldots\right)\right|+O(\varepsilon) .
\end{aligned}
$$

При этом в правой части присутствуют только тезначения $i$, для которых $\Phi_{i}\left(\Phi_{j}(x, t)\right) \neq$ $\Phi_{j}(x, t)$, т.е. $i \neq I^{\prime}(\Phi(x, t))$; если таких значений нет, то $\Phi_{j}(x, t) \in S$ и, следовательно, $(P u)\left(\Phi_{j}(x, t)\right)=g_{j}(x, t)$. Для указанных значений $i$ все $\Phi_{i}\left(\Phi_{j}(x, t)\right) \in K_{1}$, поэтому первое слагаемое в правой части не зависит от функции $u$ и, в действительности, имеет вид $\left|g_{j}\left(\Phi_{j}(x, t), \ldots, g_{i}\left(\Phi_{i}\left(\Phi_{j}(x, t)\right)\right), \ldots\right)\right|$ либо $\left|g_{j}\left(\Phi_{j}(x, t), g_{j}\left(\Phi_{j}(x, t)\right)\right)\right|$. Обозначив через $p_{2}$ наибольшую из верхних граней этих функций по всем $(x, t) \in K_{3}, j \in I$, $i \in I^{\prime}\left(\Phi_{j}(x, t)\right)$, получаем окончательно, что

$$
\left|\left(P^{2} u\right)(x, t)\right| \leqslant p_{2}+O(\varepsilon), \quad(x, t) \in K_{3},
$$

Так как $K_{1} \subseteq K_{3}$, то $p_{1} \leqslant p_{2}$.

Продолжая такие же рассуждения, получаем, что при $(x, t) \in K_{s}, s=2, \ldots, r$, имеет место оценка

$$
\left|\left(P^{s-1} u\right)(x, t)\right| \leqslant p_{s-1}+O(\varepsilon), \quad(x, t) \in K_{s},
$$

где $p_{1} \leqslant \cdots \leqslant p_{r-1}$ и все константы $p_{s}$ явно выражаются через итерации функций $\left\{g_{k}\right\}$ и $\left\{\Phi_{i}\right\}$.

Итак, в качестве числа $M$, о котором говорилось во втором абзаце доказательства теоремы 2 , можно взять любое число, большее чем $p_{r-1}$. Из проведенного рассуждения следует, что при заданном $M$ значение $\varepsilon$ можно считать произвольным из некоторого интервала $\left(0, \varepsilon_{M}\right]$. 
Зафиксируем такие $M$ и $\varepsilon=\varepsilon_{M}$ и обозначим через $Q$ множество функций $v \in C(K$, $\mathbb{R}^{n}$ ), для которых $\|v\| \leqslant M$ и $v$ в $K$ удовлетворяет условиям (4). Это непустое (в силу условия $\mathrm{У}_{3}$ ) полное метрическое пространство, и из сказанного выше следует, что каждое из отображений $P^{s}, s=1, \ldots, r-1$, переводит его в себя.

Обозначим через $L$ константу в условии Липшица, о котором говорится в условии теоремы 2 , для области $K \times\left\{u \in \mathbb{R}^{n}:|u| \leqslant M\right\}$. Тогда, взяв $v, w \in Q$, получаем

$$
\|P v-P w\| \leqslant L \max _{(x, t) \in K, i \in I^{\prime}(x, t)}\left|v\left(\Phi_{i}(x, t)\right)-w\left(\Phi_{i}(x, t)\right)\right|+2 \varepsilon L\|v-w\|
$$

(если $I^{\prime}(x, t)=\varnothing$, то $\Phi_{i}(x, t) \in S$ и потому $v\left(\Phi_{i}(x, t)\right)-w\left(\Phi_{i}(x, t)\right)=0$, т.е. такие комбинации значений $x, t, i$ из правой части исключаются).

Отсюда следует, что

$$
\begin{aligned}
\left\|P^{2} v-P^{2} w\right\| \leqslant & L \max _{(x, t) \in K, i_{1} \in I^{\prime}(x, t)}\left|(P v)\left(\Phi_{i_{1}}(x, t)\right)-(P w)\left(\Phi_{i_{1}}(x, t)\right)\right|+2 \varepsilon L\|P v-P w\| \\
\leqslant & L^{2} \max \left|v\left(\Phi_{i_{2}}\left(\Phi_{i_{1}}(x, t)\right)\right)-w\left(\Phi_{i_{2}}\left(\Phi_{i_{1}}(x, t)\right)\right)\right| \\
& +4 \varepsilon L^{2}\|v-w\|+(2 \varepsilon L)^{2}\|v-w\| ;
\end{aligned}
$$

в правой части максимум берется по $(x, t) \in K, i_{1} \in I^{\prime}(x, t), i_{2} \in\left(\Phi_{i_{1}}(x, t)\right)$ (с аналогичной предыдущему оговоркой о случае $\left.I^{\prime}=\varnothing\right)$.

Продолжая таким же образом, получаем при $s \in \mathbb{N}$ оценку

$$
\begin{aligned}
\left\|P^{s} v-P^{s} w\right\| \leqslant & L^{s} \max \left|v\left(\Phi_{i_{s}}\left(\ldots\left(\Phi_{i_{1}}(x, t)\right) \ldots\right)\right)-w\left(\Phi_{i_{s}}\left(\ldots\left(\Phi_{i_{1}}(x, t)\right) \ldots\right)\right)\right| \\
& +\varepsilon L^{s} q_{s}(\varepsilon)\|v-w\|,
\end{aligned}
$$

где $q_{s}$ - многочлен $s$-й степени, а максимум берется по $(x, t) \in K, i_{1} \in I^{\prime}(x, t), i_{2} \in$ $I^{\prime}\left(\Phi_{i_{1}}(x, t)\right), \ldots, i_{s} \in I^{\prime}\left(\Phi_{i_{s-1}}\left(\ldots\left(\Phi_{i_{1}}(x, t) \ldots\right)\right)\right)$ (с той же оговоркой).

Однако $\Phi_{i_{r-1}}\left(\ldots\left(\Phi_{i_{1}}(x, t)\right) \ldots\right) \in S$ при всех $(x, t) \in K$, и потому

$v\left(\Phi_{i_{r-1}}\left(\ldots\left(\Phi_{i_{1}}(x, t)\right) \ldots\right)\right)=w\left(\Phi_{i_{r-1}}\left(\ldots\left(\Phi_{i_{1}}(x, t)\right) \ldots\right)\right)=g\left(\Phi_{i_{r-1}}\left(\ldots\left(\Phi_{i_{1}}(x, t)\right) \ldots\right)\right)$.

Значит, при $s=r-1$ в правой части неравенства (7) первое слагаемое отсутствует. Но за счет уменьшения $\varepsilon$ (не влияющего на значение $L$ ) можно добиться выполнения неравенства $\varepsilon L^{r-1} q_{r-1}(\varepsilon)<1$. Отсюда в силу известного обобщения теоремы о сжимаюших отображениях получаем, что отображение $P$ имеет в $K$ единственную неподвижную точку, т.е. задача $(2),(4)$ имеет в $K=K_{r}$ единственное непрерывное решение $u$, для которого $\|u\| \leqslant M$.

Покажем, что для любого допустимого множества $\widetilde{K}$, для которого $\left(x^{0}, t^{0}\right) \in \widetilde{K} \subseteq K$, функция $\left.u\right|_{\widetilde{K}}$ является единственным непрерывным решением в $\widetilde{K}$ задачи $(2),(4)$. В самом деле, пусть это не так и $\widetilde{u}: \widetilde{K} \rightarrow \mathbb{R}^{n}$ является таким решением, причем $\widetilde{u}(x, t) \not \equiv(x, t)$ в $K$. Обозначим через $t^{1}$ наименьшее значение $t$, для которого $\tilde{u}(x, \tau) \equiv u(x, \tau)$ при $\tau \leqslant t,(x, \tau) \in \widetilde{K}$, а через $x^{1}$ - какую-либо точку, для которой $\tilde{u}(x, \tau) \not \equiv u(x, \tau)$ в любой близости от $\left(x^{1}, t^{1}\right)$. Тогда обе функции $\widetilde{u}$ и $u$ являются непрерывными решениями задачи $(2),(4)$ в $K_{1}:=\left\{(x, t) \in K: t \geqslant t^{1}\right\}$, для которой начальное условие при $t=t^{1}$ задается самим решением $u$. Нетрудно проверить, что условия $\mathrm{У}, \mathrm{y}_{1}, \mathrm{y}_{2}$ и $\mathrm{У}_{3}$ с заменой $\Pi$ и $\left(x^{0}, t^{0}\right)$ соответственно на $K_{1}$ и $\left(x^{1}, t^{1}\right)$ вьполняются, а так как цепочки точек $\partial K_{1}$ не могут быть длиннее, чем цепочки точек $\partial П$, то условия теоремы $2 \mathrm{c}$ той же заменой выполнены. Выбрав $M>\max \left\{\left\|\left.u\right|_{\tilde{K}}\right\|,\|\tilde{u}\|\right\}$ и применив доказанное вьше утверждение о единственности неподвижной точки, приходим к противоречию.

Теорема 2 полностью доказана. 
ЗАмЕчАниЕ. Существенность условия У для справедливости теоремы 2 очевидна. Чрезмерность требования непрерьвности функций $g_{i}$ вытекает из первого примера в п. 5; поэтому такое требование не ставится. Существенность условия У 1 не ясна; более того, автор не знает, не вытекает ли это условие из остальных требований (это относится и к условию $\mathrm{У}_{3}$, а также к условию $\mathrm{У}_{4}$ из п. 6). Условия $\mathrm{У}_{2}$ и У 3 являются вариантами известного условия согласования начальньх условий с граничными нулевого порядка (см. первый пример в п. 5).

5. Примеры. 1. Рассмотрим систему уравнений с $n=2, m=1$

$$
\frac{\partial u_{1}}{\partial t}+\frac{\partial u_{1}}{\partial x}, \quad \frac{\partial u_{2}}{\partial t}-\frac{\partial u_{2}}{\partial x}=0
$$

в области П: $0 \leqslant x<\infty, 0 \leqslant t<\infty$. Здесь $S_{1}=\{(0, t): 0<t<\infty\} \cup\{(x, 0): 0 \leqslant x<\infty\}$, $S_{2}=S=\{(x, 0): 0 \leqslant x<\infty\} ; I(0,1)=\{1\}(0<t<\infty), I(x, 0)=\{1,2\}(0 \leqslant x<\infty)$. Добавочные условия (4) имеют общий вид:

$u_{1}(0, t)=g_{1}\left(0, t, u_{2}(0, t)\right) \quad(0<t<\infty), \quad u_{j}(x, 0)=g_{j}(x, t) \quad(0<x<\infty, j=1,2)$.

Условие $\mathrm{У}_{3}$ означает непрерывность функций $x \mapsto g_{j}(x, 0), 0 \leqslant x<\infty, j=1,2$, а также существование непрерывной функции $t \mapsto h(t) \quad(0 \leqslant t<\infty)$, для которой $h(0)=g_{2}(0,0)$, а функция $t \mapsto g_{1}(0, t, h(t)) \quad(0<t<\infty)$ непрерывна и стремится $\mathrm{K}$ $g_{1}(0,0)$ при $t \rightarrow 0^{+}$. Отсюда, в частности, вытекает хорошо известное условие согласования: $g_{1}\left(0,0, g_{2}(0,0)\right)=g_{1}(0,0)$, в котором левая часть понимается как предел. Из условия $\mathrm{У}_{2}$ вытекает, в частности, инвариантность условия согласования относительно отображения $P$, определенного формулой (6).

В применении к системе (8) для вьполнения условий теоремы 2 достаточно (и, по-видимому, необходимо), чтобы каждая из задаваемых функций $x \mapsto g_{j}(x, 0), 0 \leqslant x<\infty$, $j=1,2$, и $(t, s) \mapsto g_{1}(0, t, s), 0<t<\infty, s \in \mathbb{R}$, была непрерывной, последняя функция допускала непрерьвное продолжение на $[0, \infty) \times \mathbb{R}$ и выполнялось указанное выше условие согласования. Однако априорное требование непрерьвности функции $g_{1}$ на $S_{1}$ привело бы к равенству $g_{1}\left(0,0^{+}, s\right) \equiv g_{1}(0,0)$. В частности, если $\left.g_{1}\right|_{x=0}$ не зависит от $t$, то эта величина не должна зависеть и от $s$, т.е. она должна быть постоянной. Ясно, что это требование не является естественным для рассматриваемой задачи.

2. Рассмотрим систему уравнений (8) в области П: $0 \leqslant x \leqslant t^{2}, 0 \leqslant t \leqslant 1 / 2$ при добавочных условиях

$$
u_{1}(0, t)=(1-t) u_{2}(0, t), \quad u_{2}\left(t^{2}, t\right)=u_{1}\left(t^{2}, t\right), \quad 0 \leqslant t \leqslant \frac{1}{2}, \quad u_{1}(0,0)=u_{2}(0,0)=1 .
$$

Ясно, что здесь длины цепочек неограничены, тогда как прочие условия теоремы 2 выполнены. Покажем, что поставленная задача не имеет непрерывного решения ни в какой окрестности точки $(0,0)$.

В самом деле, пусть решение $u$ определено при $0 \leqslant x \leqslant t^{2}, 0 \leqslant t \leqslant h(\leqslant 1 / 2)$. Обозначим через $а$ значение функции $u_{1}$ в какой-либо точке $\left(0, t_{0}\right), 0<t_{0} \leqslant h$. Тогда значение функции $u_{2}$ вдоль характеристики 2-го семейства с уравнением $t=t_{0}-x$ равно $a\left(1-t_{0}\right)^{-1}$. Эта характеристика начинается в точке ее пересечения с правой границей области П, т.е. в точке $M\left(\left[1+2 t_{0}-\left(1+4 t_{0}\right)^{1 / 2}\right] / 2,\left[-1+\left(1+4 t_{0}\right)^{1 / 2}\right] / 2\right)$. Таким образом, $u_{2}(M)=a\left(1-t_{0}\right)^{-1}$. Теперь рассмотрим характеристику первого семейства, 
заканчивающуюся в точке $M$. В доль нее значение функции $u_{1}$ постоянно и равно $u_{2}(M)$. Эта характеристика начинается в точке $\left(0, t_{1}\right)$, где $t_{1}:=\left(1+4 t_{0}\right)^{1 / 2}-1-t_{0}$. Таким образом, $u_{1}\left(0, t_{1}\right)=a\left(1-t_{0}\right)^{-1}$.

Затем, проделав такую же процедуру для точки $\left(0, t_{1}\right)$, получаем точку $\left(0, t_{2}\right)$ и значение $u_{1}\left(0, t_{2}\right)$ и т.д. Продолжая таким образом, мы получим убьвающую последовательность значений $t_{j}>0$ и соответствующую последовательность значений компоненты $u_{1}$ решения, определяемые рекуррентными соотношениями

$$
t_{j+1}=\left(1+4 t_{j}\right)^{1 / 2}-1-t_{j}, \quad u_{1}\left(0, t_{j+1}\right)=u_{1}\left(0, t_{j}\right)\left(1-t_{j}\right)^{-1}, \quad j=0,1, \ldots
$$

Докажем, что $\prod_{j=0}^{\infty}\left(1-t_{j}\right)^{-1}=\infty$ и тем самым придем к противоречию с условием $u_{1}(0,0)=1$. Так как $1 / 2 \geqslant t_{j} \rightarrow 0$ при $j \rightarrow \infty$, то достаточно проверить, что $\sum_{j=0}^{\infty} t_{j}=\infty$. Для доказательства покажем, что $t_{j} \geqslant \tau_{j}:=p /(j+1)$ при всех $j=0,1, \ldots$ и $p:=\min \left\{t_{0}, 1 / 16\right\}$. В самом деле, из рекуррентного соотношения для $t_{j}$ следует, что $\mid \Delta t_{j}\left(:=\left|t_{j+1}-t_{j}\right|\right)=8 t_{j}^{2}\left[1+\left(1+4 t_{j}\right)^{1 / 2}\right]^{-2}<8 t_{j}^{2}$, тогда как $\left|\Delta \tau_{j}\right|=$ $(j+1)[p(j+2)]^{-1} \tau_{j}^{2}$. Неравенство $t_{0} \geqslant \tau_{0}$ очевидно. Поэтому, если указанная оценка $t_{j}$ неверна, то существует значение $j=r \geqslant 0$ такое, что $t_{r} \geqslant \tau_{r}$, но $t_{r+1}<\tau_{j+1}$. Отсюда $\left|\Delta t_{j}\right|>\left|\Delta \tau_{j}\right|$, а потому $8 t_{r}^{2}>\left|\Delta t_{r}\right|>\left|\Delta \tau_{r}\right| \geqslant(2 p)^{-1} \tau_{r}^{2} \geqslant(2 p)^{-1} t_{r}^{2}$ и мы вступаем в противоречие с определением числа $p$.

3. Рассмотрим систему уравнений с $n=4, m=2$

$$
\begin{aligned}
\frac{\partial u_{1}}{\partial t}-\frac{\partial u_{1}}{\partial x_{1}}=f_{1}(x, t, u), & \frac{\partial u_{2}}{\partial t}-\frac{\partial u_{2}}{\partial x_{2}}=f_{2}(x, t, u), \quad \frac{\partial u_{3}}{\partial t}+\frac{\partial u_{3}}{\partial x_{1}}=f_{3}(x, t, u), \\
\frac{\partial u_{4}}{\partial t}+\frac{\partial u_{4}}{\partial x_{2}} & =f_{4}(x, t, u)
\end{aligned}
$$

в области $\Pi:\left\{(x, t): x_{1}^{2}+x_{2}^{2} \leqslant 1,0 \leqslant t<\infty\right\}$. Здесь $S_{1}=\left\{(x, 0): x_{1}^{2}+x_{2}^{2} \leqslant 1\right\} \cup\{(x, t)$ : $\left.x_{1}^{2}+x_{2}^{2}=1, x_{1} \geqslant 0,0<t<\infty\right\}$, а $S_{2}, S_{3}$ и $S_{4}$ получаются из $S_{1}$ последовательньми поворотами на угол $\pi / 2$ вокруг оси $t$ в положительном направлении. Этим определяются аргументы функций $g_{j}$.

Для дальнейшего обозначим через $D_{1}\left(D_{2} ; D_{3} ; D_{4}\right)$ полосы $\left\{(x, t): x_{1}^{2}+x_{2}^{2}=1, x_{1}>0\right.$, $x_{2}>0$ (соответственно $\left.\left.x_{1}<0, x_{2}>0 ; x_{1}<0, x_{2}<0 ; x_{1}>0, x_{2}<0\right), 0<t<\infty\right\}$, a через $\bar{D}_{j}$ - замыкание множества $D_{j}$. Тогда для выполнения условий теоремы 2 достаточно (и, по-видимому, необходимо), чтобы

1) все функции $x \mapsto g_{j}(x)$ были непрерывными;

2) функции $\left(x, t, s_{3}, s_{4}\right) \mapsto g_{j}\left(x, t, s_{3}, s_{4}\right), j=1,2$, допускали непрерывное продолжение с $D_{1}$ на $\bar{D}_{1}$, и на $\bar{D}_{1} \cap S$ выполнялись условия согласования: $g_{j}\left(x, 0, g_{3}(x)\right.$, $\left.g_{4}(x)\right)=g_{j}(x), j=1,2$; аналогичные требования вьполнялись в $D_{2}, D_{3}$ и $D_{4}$;

$3)$ на луче $(1,0, t), 0 \leqslant t<\infty$, функции $\left(t, s_{2}, s_{3}\right) \mapsto g_{1}\left((1,0), t, s_{2}, s_{3}\right)$ и $\left(t, s_{3}, s_{4}\right) \mapsto$ $g_{1}\left((1,0), t, s_{3}, s_{4}\right)$ совпадали и потому первая не зависела от $s_{4}$, а вторая - от $s_{3}$; аналогичные требования выполнялись для лучей $(0,1, t),(-1,0, t)$ и $(0,-1, t)$, $0 \leqslant t<\infty$.

6. Нелокальные утверждения. В этом пункте будем без особой оговорки считать, что выполнены все предположения теоремы 2 , причем условия У 1 и У 2 справедливы для любой точки $\left(x^{0}, t^{0}\right) \in S$, и, кроме того, выполнено следующее условие.

$\mathrm{Y}_{4}$. Для любой точки $\left(x^{0}, t^{0}\right) \in \Pi$ существует содерхащее ее ограниченное допустимое множество. 
ТЕОрема 3. Пусть непрерывные решения задачи (2), (4) существуют в допустимых мнохсествах $K_{1}$ и $K_{2}$. Тогда в $K_{1} \cap K_{2}$ әти решения совпадают.

ДокАЗАтЕЛьство. Обозначим упомянутые решения соответственно через $u^{1}$ и $u^{2}$, и пусть $u^{1}\left(x^{0}, t^{0}\right) \neq u^{2}\left(x^{0}, t^{0}\right)$, где $\left(x^{0}, t^{0}\right) \in K_{1} \cap K_{2}$. Выберем на основе условия $\mathrm{У}_{4}$ ограниченное допустимое множество $K$ так, что $\left(x^{0}, t^{0}\right) \in K$; в силу условия У можно считать $K$ замкнутьм. Теперь получение противоречия не отличается от проведенного в последнем абзаце доказательства теоремы 2 .

Назовем непрерывное решение $u: D \rightarrow \mathbb{R}^{n}$ задачи (2), (4) непродолэсимым, если $D$ открыто в П и $u$ является расширением любого другого непрерьвного решения той же задачи с открытой в П областью определения. (При $m=1$ такое решение было исследовано в докладе [5].)

Теорема 4. Непродолжимое непрерывное решение $u: D \rightarrow \mathbb{R}^{n}$ задачи (2), (4) существует и единственно, причем $S \subseteq D$.

ДокАЗАТЕЛЬСТво. В качестве $D$ - области определения искомого решения - возьмем объединение всех открытых в П областей определения непрерьвных решений задачи (2), (4). В силу теоремы 3 все эти решения определяют на $D$ единую функцию, которая, очевидно, и является единственным непрерывным непродолжимым решением задачи (2), (4). Включение $S \subseteq D$ очевидно.

Следующая теорема говорит о поведении непродолжимого непрерьвного решения $u$ вблизи точек границы его области определения, внутренних для П.

ТЕорема 5. Пусть точка $\left(x^{0}, t^{0}\right)$ содержится в замыкании множества $\partial D \backslash \partial П$, и предел при $k \rightarrow \infty$ любой сходящейся последовательности вида $\left(x^{0}-x^{k}\right) /\left(t^{0}-t^{k}\right)$, где $t^{k} \rightarrow\left(t^{0}\right)^{-}, x^{k} \rightarrow x^{0},\left(x^{k}, t^{k}\right) \notin D$ (если такие последовательности существуют), не принадлежит выпуклой оболочке множества $\left\{\lambda_{1}\left(x^{0}, t^{0}\right), \ldots, \lambda_{n}\left(x^{0}, t^{0}\right)\right\}$. Тогда решение и не ограничено при $t<t^{0}$ в пересечении $D$ с любой окрестностью точки $\left(x^{0}, t^{0}\right)$.

ДокАЗАТЕЛЬСТво. Пусть условия теоремы 5 выполнены, но ее утверждение неверно. Будем сначала считать, что $\left(x^{0}, t^{0}\right) \notin \partial \Pi$, и обозначим через $A$ вьпуклую оболочку, упомянутую в формулировке теоремы, а через $A_{p}$ замыкание ее $p$-окрестности $(p>0)$. Зафиксируем $p$ столь мальм, что для множества $A_{p}$ справедливо свойство, указанное для $A$ в формулировке теоремы. Обозначим, далее, через $B_{h}(h>0)$ замкнутьй конус с вершиной в точке $\left(x_{0}+h^{2} a, t_{0}+h^{2}\right)$ и основанием $C_{h}:=\left(x_{0}-h A_{p}, t_{0}-h\right)$, где $a \in A-$ фиксированная точка.

Ясно, что если $h$ достаточно мало, то $B_{p} \subset \Pi, C_{h} \subset D$, а все характеристики с концевыми точками в $B_{h}$, продолженные в сторону убывания $t$, достигают $C_{h}$, не вьходя из $B_{h}$. Поэтому задача $(2),(4)$, рассмотренная в $B_{h}$, содержит только начальные условия, поставленные при $t=t_{0}$, т.е. это задача Коши. Будем считать, что начальные условия задаются изучаемым решением $u$. Тогда, пользуясь допущением об ограниченности $u$ в некоторой окрестности точки $\left(x^{0}, t^{0}\right)$ при $t<t_{0}$, с помощью стандартных рассуждений получаем существование непрерывного решения задачи $(2),(4)$ в $B_{h}$, если $h$ достаточно мало́. Но точка $\left(x^{0}, t^{0}\right)$ является внутренней для конуса $B_{h}$. Поэтому мы получаем продолжение непрерьвного решения задачи $(2),(4)$ на некоторую полную окрестность точки $\left(x^{0}, t^{0}\right)$, что противоречит предположению о $\left(x^{0}, t^{0}\right) \in \partial D$. 
Случай, когда $\left(x^{0}, t^{0}\right) \in \partial \Pi$, рассматривается аналогично, но в качестве множества $B_{h}$ надо взять пересечение описанного вьше конуса с $\Pi$, а задача $(2),(4)$ в $B_{h}$ становится начально-граничной. После переноса начального условия на основание конуса множество $B_{h}$ для полученной задачи оказьвается допустимым. В остальном схема рассуждений аналогична указанной вьше и следует доказательству теоремы 2.

Теорема 5 доказана.

ТЕОРема 6. Пусть условие Липиииа, о котором говорится в теореме 2, является глобальным, а при любом $t \in \mathbb{R}$ множество $\Pi \cap\{(x, \tau): \tau \leqslant t\}$ ограниченное. Тогда $D=\Pi$ (см. теорему 4).

ДокаЗАТЕЛЬСтво. Пусть $u: D \rightarrow \mathbb{R}^{n}$ - непродолжимое непрерывное решение задачи $(2),(4)$, причем $D \neq \Pi$. Положим $t_{0}:=\min \{t:(\forall x:(x, t) \in D)\}, T:=\min \{t:(\forall x:$ $(x, t) \in \Pi \backslash D)\}, U(t):=\min \{|u(x, t)|:(x, t) \in D\}, t_{0} \leqslant t<T$. Тогда $-\infty<t<T<\infty$ и из теоремы 5 следует, что $U(t) \rightarrow \infty$ при $t \rightarrow T-$. Покажем, что последнее невозможно.

В самом деле, применяя при $(x, t) \in D, t<T$ условие Липшица и учитьвая локальную ограниченность функций $g_{i}$ и $f_{i}$ получаем, что

$$
\left|u_{i}(x, t)\right| \leqslant K_{1}+L \max _{j}\left|u_{j}\left(\Phi_{i}(x, t)\right)\right|+L \int_{\chi_{i}(x, t)}^{t} \max _{j}\left|u_{j}\left(\varphi_{i}(\tau ; x, t), \tau\right)\right| d \tau,
$$

где $L$ - константа в условии Липшица, а $K_{1}$ - постоянная, зависящая только от значения $T$. Применим к $u_{j}$ в правой части такую же оценку, а затем проведем итерацию этой процедуры. Учитывая локальную ограниченность длин цепочек в П, мы через конечное число шагов придем к неравенству вида

$$
\left|u_{i}(x, t)\right| \leqslant K_{r}+L^{r} \int_{t_{0}}^{t} U(\tau) d \tau \Rightarrow U(t) \leqslant K_{r}+L^{r} \int_{t_{0}}^{t} U(\tau) d \tau .
$$

Применяя к последнему неравенству лемму Гронуолла, получаем ограниченность функции $t \mapsto U(t)$ при $t<T$, т.е. противоречие с нашим предположением.

Теорема 6 доказана.

Выражаю благодарность А. М. Филимонову за литературные указания.

\section{СПИСОК ЦИТИРОВАННОЙ ЛИТЕРАТУРЫ}

[1] Курант Р. Уравнения с частными производными. М.: Мир, 1964.

[2] Аболиня В. Э., Мышкис А. Д. Смешанная задача для почти линейной гиперболической системы на плоскости // Матем. сб. 1960. Т. 50. № 4. С. 423-442.

[3] Cesari L. A boundary-value problem for quasilinear hyperbolic systems // Riv. Nat. Univ. Parma. 1974. V. 3. № 3. P. 107-131.

[4] Cesari L. A boundary-value problem for quasilinear hyperbolic systems in the Schauder canonic form // Ann. Squola Norm. Sup. Pisa. 1974. V. 4. №1. P. 311-358.

[5] Мышкис А. Д. О максимальной области разрешимости смешанной задачи для почти линейной гиперболической системы с двумя независимыми переменными // Материалы к совместному советско-американскому симпозиуму по уравнениям с частными производными. Новосибирск: СО АН СССР, 1963. С. 1-10. 